\title{
A Process-Centric Approach for Teaching Digital Fabrication
}

\author{
Francisco Valdes \\ Georgia Institute of Technology, USA \\ fvaldes6@gatech.edu
}

\author{
Andres Cavieres \\ Georgia Institute of Technology, USA \\ andres.cavieres@gatech.edu
}

\author{
Russell Gentry \\ Georgia Institute of Technology, USA \\ russell.gentry@coa.gatech.edu
}

\begin{abstract}
New generation of fabrication machines, such as 3-D printers, do not use "real" materials nor processes generally used in building construction, and so further exacerbate the disconnect between laboratory-based prototyping and full-scale building construction techniques. This research critically reviews the results of a graduate fabrication course from a process-centric standpoint. Students in the course create specification for fabrication and assembly activities through a diagrammatic language that integrates several types of construction knowledge such as design, material properties, machine constraints, and assembly guidelines. This document presents the foundations of the methodology, and discussed the results based on two criteria: Process Modeling and CAD/CAM workflow.
\end{abstract}

Keywords: Process model; CNC, Fabrication, Parametric modeling, CAM.

\section{Introduction}

Recent approaches for teaching digital fabrication in Architecture and Industrial Design have concentrated mostly on the integration of digital and parametric modeling with CNC equipment and 3D prototyping technologies. While these approaches expose the students to the most advanced digital technologies, they lack a more comprehensive perspective on the role that these technologies play in the context of current industry processes and constraints. In particular, the complexities associated with the integration of multiple material systems, the selection criteria for a given fabrication method and the sequential logic required for assembly are seldom taken in consideration. While a parametric model can be easily modified, allowing multiple design alternatives, many students do not know why, when and how to establish the parametric relationships that are relevant for given a design problem. This lack of understanding is due, partially, to their lack of exposure to real construction materials and fabrication techniques. To address this problem, the paper describes an introductory graduate course developed in a fabrication laboratory within the School of Architecture at the Georgia Institute of Technology. The course titled "Construction Materials, Systems, and Fabrications" provides an introduction to fabrication in a variety of commonly-encountered construction materials. The course is open to graduate students in Architecture and Industrial Design, and its main focus is on a process-driven relationships between geometry, material affordances, fabrication techniques and construction. Besides giving the students the opportunity to have a hands-on experience with a series of fabrication techniques, the course emphasizes representational aspects of designs that enable the integration of fabrication and construction considerations early in the design process. Particularly, this goal is achieved through the integration of geometric models with the description of fabrication and construction activities represented in process models.

\section{Background}

\section{Representation: Parametric modeling and process modeling}

Since 1970's, there have been tremendous advances and flexibility in the creation of geometric models through CAD tools. However, these geometric models still do not provide much context about real construction materials and processes. Lee et al. (2006) explored the potential of embedding design and engineering knowledge in production software for building information modeling (BIM). They developed the Building Object Behavior (BOB) description notation for describing the connections and assembly of precast concrete objects. Lyon (2007) proposed a method for embedding fabrication knowledge regarding CNC milling processes with a design tool that facilitates the production of machine tiles with consistent boundaries across the tiles. Cavieres et al. (2011) analyze the prospective uses of parametric modeling to embed construction and structural design knowledge in the form of generative rules and feedback rule-checking functions - and applied these rules to the design of load-bearing masonry buildings. The objective of their work was to tie knowledge of constructive and structural principles during conceptual design in order to improve early decision-making.

Another relevant area of research is the study of design process in engineering. Murata (1988), reviews the Petri Nets ${ }^{1}$ history and application areas, and specifically describes process systems that are concurrent, asynchronous, distributed, parallel, nondeterministic, and/or stochastic. He also describes how Petri Nets approach can be used as a graphical tool to create flowcharts, block diagrams, and networks. Bucciarelli (1998), reports studies of the design process within engineering firms. The studies were

\footnotetext{
${ }^{1}$ Petri nets or place/transition nets are a form of graph diagram used to describe the position of and flow of objects or information through a process.
} 
based on participant-observation techniques: in each case the firm was approached in the way an ethnographer might approach a foreign culture. Motte (2008) explains how in an overall perspective, systematic engineering design process models constitute the core upon which concurrent product development models are built. The structure that these systematic models give to the engineering design process and their inherent logic explain in part their success. Conway et al. (2012) charts the development of a system architecture designed to address the challenges associated with creating accurate and re-usable records of synchronous design activities. The common theme in this engineering design literature is that flows of information, flows of material, and even the development of a design are processes, which can be modeled and managed. This idea of a "process model" is absent in much of our architectural teaching, and in our opinion is core to the teaching of digital fabrication.

\section{Hands-on/Hands-off fabrication}

Lyon (2007) explores new methods to integrate manufacturing processes information into design phases. By means of design for manufacturing (DfM) concept, and looking at relations between its potential applications in architectural production, his Ph.D. thesis implemented a DfM model that varies from previous models by incorporating learning in the process. Celani (2012), discusses the evolution of experimental fabrication in architecture. Celani goes on to describe approaches to architectural teaching as either "scientific method or inquiry" or "guided instruction". The teaching of digital fabrication within the studio has the goal of design production, where students develop their own objectives and inquires and the discussion therefore focuses on the designed product and not on methods, models, and processes. In contrast, a course on fabrication - with an attenuated emphasis on design - is closer to Celani's definition of "guided instruction". In this case, the pedagogy tries to reduce the cognitive load for the students, while giving them the time and space to learn a wide variety of fabrication machines. Celani states that architecture curricula often reject the model of guided instruction as being too vocational, perhaps diminishing the value of design. We support the ideals of guided instruction within the teaching of digital fabrication, as gives us headroom to teach not only the technical aspects of digital modeling and CNC translation, but also the relationships between material and process, process and assembly, and specific aspects of construction in multiple material systems such as dimensional tolerancing.

\section{Approach}

The course's primary goal is to tie knowledge of construction materials to techniques for fabrication, and subsequently to an understanding of fabrication processes and machines. Students work in four major classes of construction materials: wood, concrete, thermoplastic polymers and metals. In the course, the materials and construction systems are covered at two different levels: at a component level where different parts of an assembly are developed in one material, by means of a single fabrication technique (either manual or digital fabrication); and an assembly level, where the different components are aggregated and connected into a final assembly.

\section{Specific objectives of the course are described as follows:}

- Reiterate, reinforce and extend knowledge on basic material properties and construction techniques for common materials used in building construction - from what is assumed to be a basic knowledge from undergraduate construction technology and structures courses.

- Develop a knowledge structure that ties material fabrication techniques to material properties and form.

- Introduce notational means for describing parts creation and component assembly through process models.

- Extend this notation into computational frameworks for digital representation of fabrications and processes.

- Introduce underlying file formats for communication with digital fabrication machines (g-code, STL).

- Use high-level CAD/CAM software for creation of fabrication instruction sets, and complete a series of tightly-constrained design exercises using commercial CAD/CAM software.

- Receive hands-on experience with material processing, from design representation and specification to machine operation and assembling.

\section{Curtain wall assembly}

The laboratory exercises taken together focus on the production of what is described as a curtain wall assembly, at approximately one-quarter scale. Each student produces one laminated wood column; two tiles or "panels", one of UHPC (ultra high performance concrete) and one of thermoformed plastic; and the steel connections necessary to connect all of the components (Table 1). Additional intermediate components such as molds, jigs and fixtures are produced in order to achieve the final assembly. During the semester, the students are required to think ahead to the final assembly, coordinating their components in their own final assembly as well as with the students having adjacent projects. 
Table 01: Final assembly components and key material/processes description

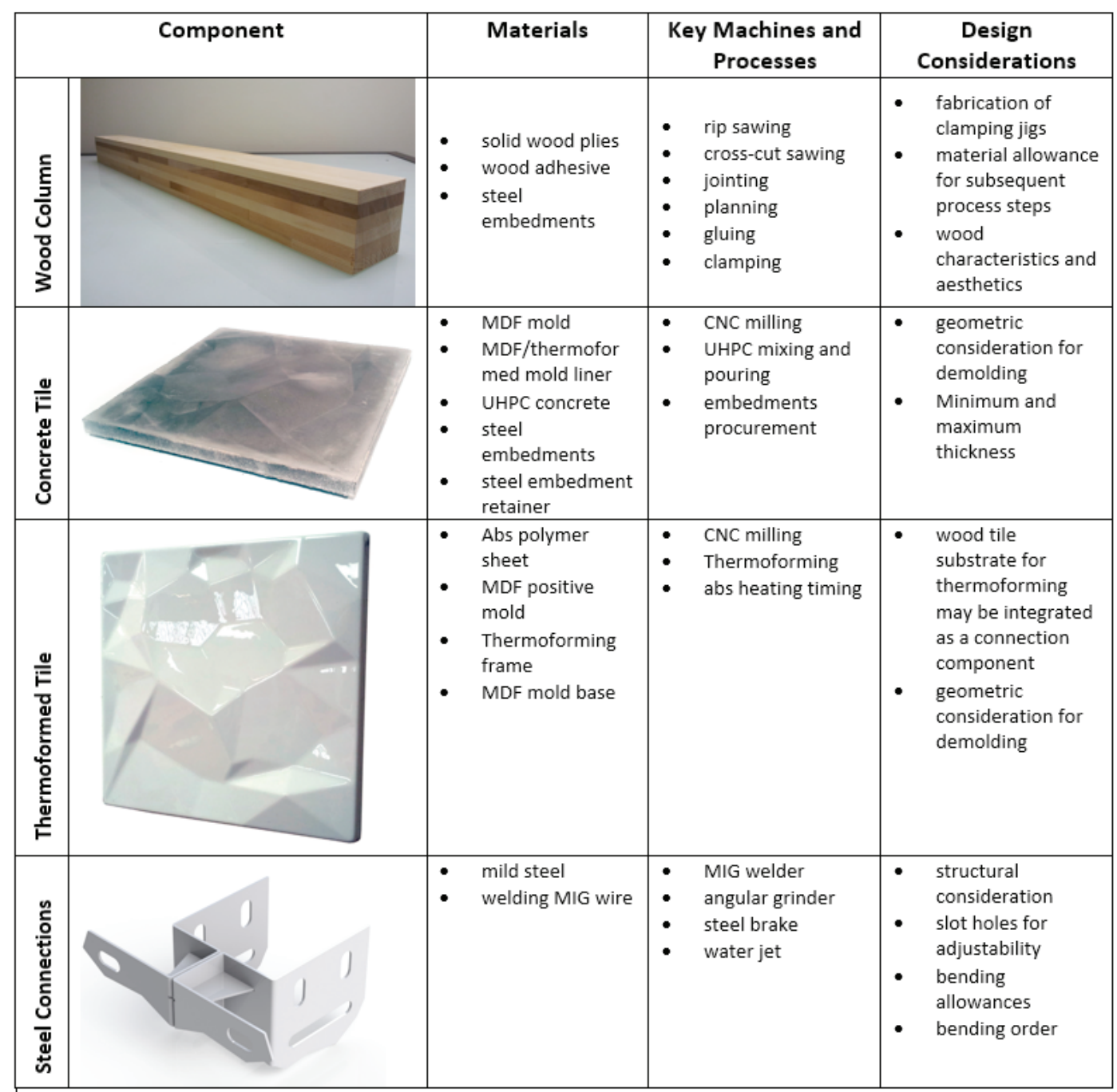

\section{Process modeling}

Students in the course create detailed a specification for fabrication and assembly activities by means of a diagrammatic language that integrates multiple sources of construction knowledge such as design intent, material properties, machine parameters, and assembly instructions. This diagrammatic language was specially developed for the course, based on the syntactical structure of conceptual maps, using CMAP tools, an open source conceptual modeling tool ${ }^{2}$.

The semantics of our language is based on principles derived from Petri Nets, Business Process Modeling Notation (BPMN) and SysML activity diagrams - but in a simplified free-form flowchart format that the students can easily understand and adapt $^{3}$. The key

${ }^{2}$ CMAP Tools. Institute for Human and Machine Cognition (IHMC). http://cmap.ihmc.us/

${ }^{3}$ We have found that masters-level design students, in contrast to research students studying at the doctoral level, have limited patience with the intricacies and semantic rigor of process model notations such as BPMN and Sys-ML activity diagrams. The CMAP is considered to be a compromise semantic characteristic of the notation is the description of a set of necessary pre-conditions and post-conditions that students need to postulate and represent prior any fabrication activity. Post-conditions describe the goal of a given fabrication operation, meaning the geometric and material transformation of the designed parts and assemblies. The pre-conditions describe the different types of information that are necessary to perform a fabrication machine operation correctly, including design geometry, material information (e.g. stock, dimensions, hardness), machine parameters (e.g. machine tolerances, fixtures, machine feed and speed, type of lubrication, etc.), as well as safety procedures. The CMAP (Fig.01) represents intermediate work products (such as the production of plies for the laminated wood columns) so that the requirements for the part can be considered in a rational step-wise process.

- more formal than a flowchart but without the abstraction and rigorous syntax found in more formal process modeling graphs. 


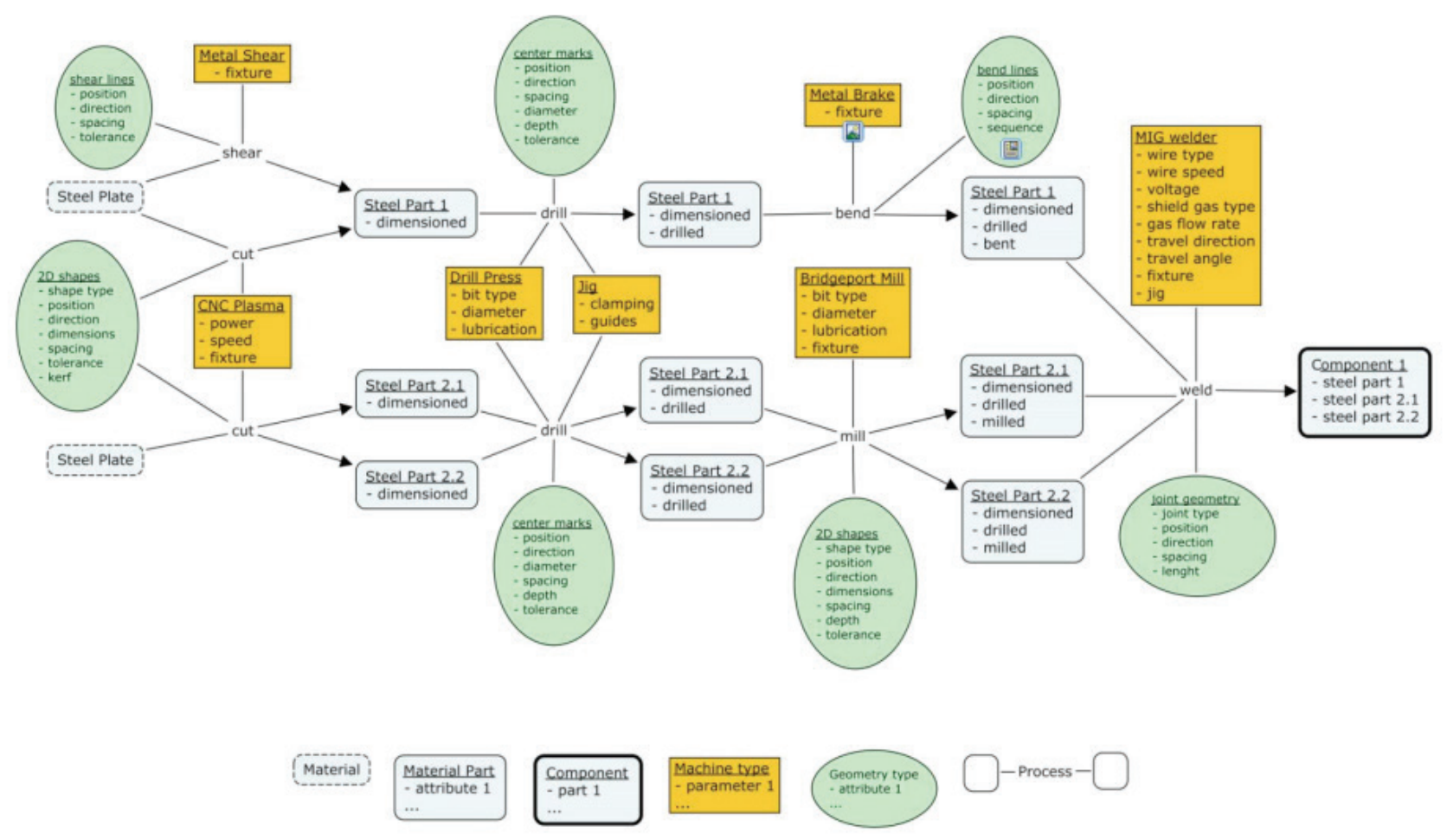

Figure.1: Example of the CMAP process model from the course

\section{Results and Discussion}

The following figure (Fig.02) shows an example of steps and deliverables for each course assignment. At first, the students have an assignment handout with the specific details and dimensional considerations for the component to be fabricated (1). Then, the students design and develop CAD representations of the component (2). This step is meant to be simple because the focus is mainly on fabrication. The third step (3) requests the students to suggest a process model for CAD/CAM workflow and for fabricating the component and to express this workflow in the process model. Finally, the students fabricate their components and critically review the a priori fabrication approach and revise their process models to represent the as-fabricated condition. The results and discussion that follows has been divided in two main categories: Process models and CAD/CAM workflow.

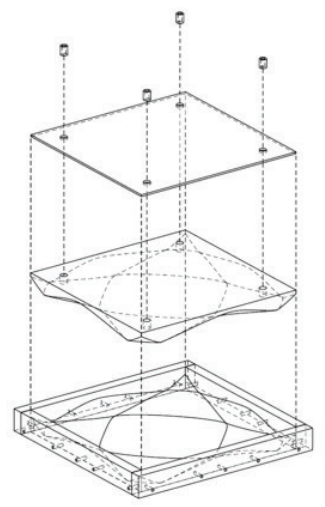

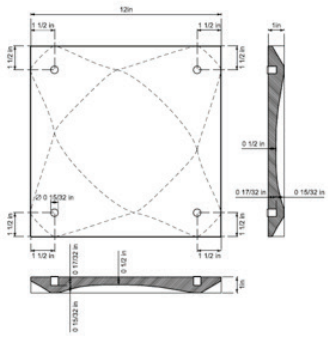

1
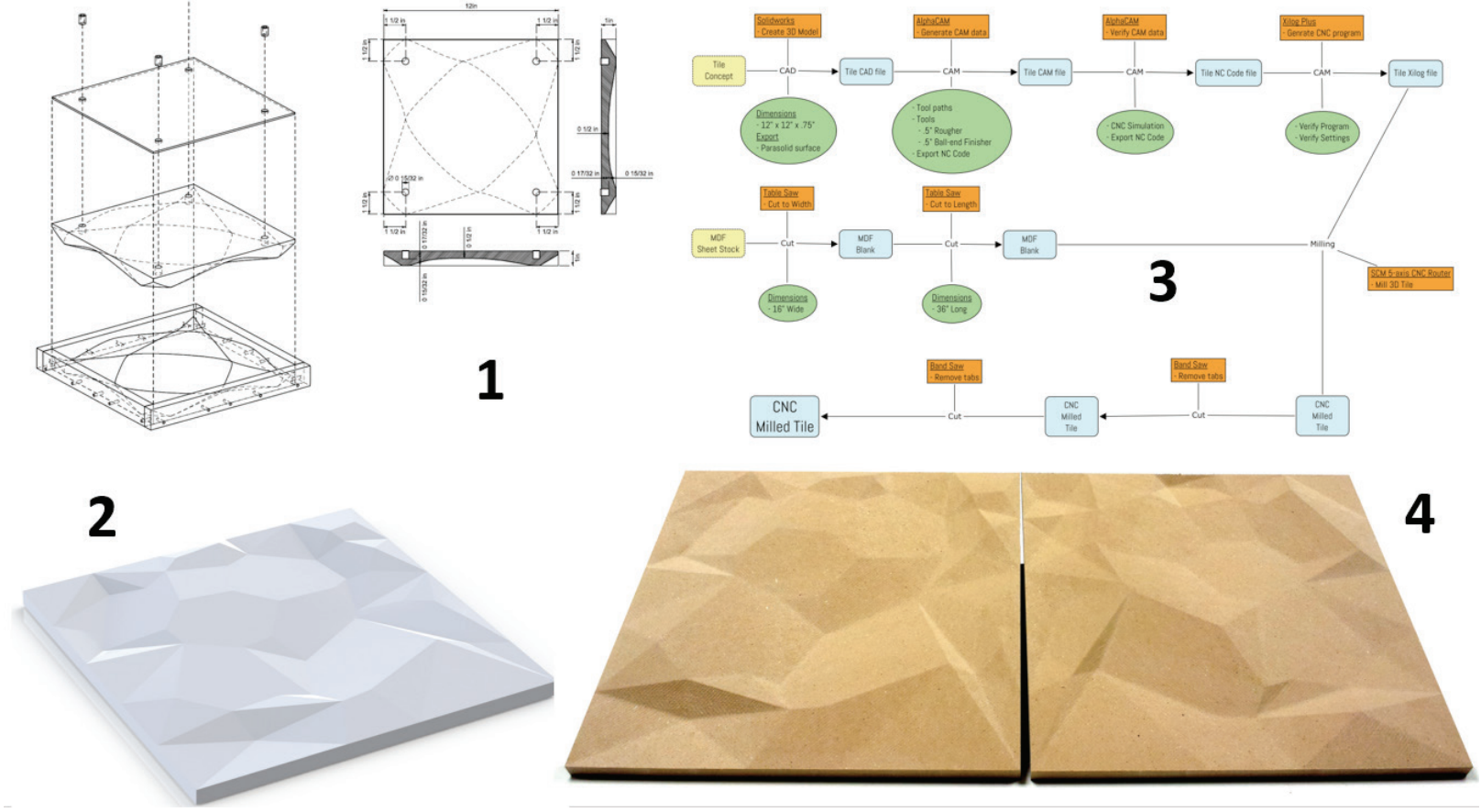

Figure.2: Main stages of the course assignments 


\section{Process models: documentation the development of fabrication knowledge}

For each assignment, the students develop 2 process models. The before and after process models speak to different pedagogical objectives. The before model is a bit pragmatic, as a way of assessing whether the student is capable of producing the proposed part or is missing significant aspects of the exercise. In this sense the $a$ priori process model is diagnostic - often exposing the students' naiveté. Nonetheless, the "after" provides the feedback loop for self-correction and an opportunity to size the impact of design/fabrication decisions.

A central consideration is the expressiveness of the graphical language that students use to describe their processes. Sometimes, they extended the language to represent some "unsupported" activities and processes. However, this individual assessment was a source of semantic differences natural of such customizations. Although we agree that the structure and semantics of the provided graphical language can be extended and improved, the primary goal is not to teach process modeling languages creation, nor to focus on consistency and reusability of process models. One addition that is missing, based on our experience with BPMN and SysML activity diagrams, is the use of decision gates (which lead to loops). Other than that, we consider that the language may lose its simplicity and main pedagogical purpose.

\section{CAD/CAM workflow}

The decision to allow or require the use of fully-parametric CAD tool, in this introductory level course, has been a matter of long discussion in our group. In our research, we have found that the use of such tools greatly facilitates the process and documentation of a given building or product. However, modeling with featurebased solid modeling tools requires the user to specify relations and constraints at two different levels: internal relations, which are necessary for computational consistency of the model; and external relations, which are required for keeping the consistency of the model with real world laws. Our course focuses primarily in the second kind of relationship. Students entering the course with little or no parametric modeling expertise have more difficulty establishing parametric relations for external consistency. This problem arises from their lack of hands-on experience with materials, tools and machines. This is the core argument of the course, and one reason behind using non-parametric CAD system. Almost all of our students have worked with non-parametric tools such as Rhinoceros and AutoCAD. In our teaching we have highlighted the limitations that "pure geometry" tools such as these generate in the fabrication process, and we have introduced simple parametric modeling approaches using some parametric tools with feature-based design modules such as Solidworks, Siemens NX, and Autodesk Inventor. In particular, with simple components that carry just a few geometric constraints, these CAD packages have been successful in getting the students started in the field of parametric modeling.
Another important part of the design/fabrication process is CAD/CAM interoperability. In the CAM side, for most of the assignments, the course focuses in learning AlphaCAM. This application offers robust options for importing CAD geometry from different CAD sources. Here, the students also obtain their GCode, which is a basic language to translate solid geometry to a set of machine readable manufacturing operations. In addition, by means of AlphaCAM, the students frame their Process Models matching the order of operations as they are shown in the application GUI.

\section{Conclusions}

This paper presented a novel process-centric approach for teaching fabrication within the school of architecture. It discusses the role of process modeling for fabrication at an introductory level, and suggests what needs to be addressed in more advanced digital fabrication classes. Starting from our assumption that architectural design ideas from novice designers often lack knowledge about buildings materials and assembly activities, the course is taught in the context of real construction techniques, not just at a prototype or model level. Thus, understanding and modeling fabrication processes promotes the capture and reusability of design knowledge while helping novice designers to create more practicable fabrication projects. Future development of this research will compare the impact of parametric modeling vs. non parametric modeling in teaching fabrication, and will also propose a methodology to integrate the present approach with traditional design activities of the design studio.

\section{Bibliography}

Cavieres, A. Gentry, R. Al-Haddad, T (2011). Knowledge-based parametric tools for concrete masonry walls: Conceptual design and preliminary structural analysis, Automation in Construction, Volume 20, Issue 6, Pages 716-728,

Lyon, E. (2007). Component based design and digital manufacturing cm: a design for manufacturing model for curved surfaces fabrication using three axes computer numerical controlled router. Ph.D. dissertation, Georgia Institute of Technology.

Murata, T (1989). Petri nets: properties, analysis and applications Proc. IEEE, 77 (4) pp. 541-580

Bucciarelli, L. L. (1988). "Ethnographic perspective on engineering design." Design Studies 9(3): 159-168.

Ghang Lee, Rafael Sacks, Charles M. Eastman (2006). Specifying parametric building object behavior (BOB) for a building information modeling system, Automation in Construction, Volume 15, Issue 6, Pages 758776 ,

Celani, G. (2012). "Digital Fabrication Laboratories: Pedagogy and Impacts on Architectural Education." Nexus Network Journal 14(3): 469-482.

Motte, D. (2008). A review of the fundamentals of systematic engineering design process models. 10th International Design Conference, DESIGN 2008, May 19, 2008 - May 22, 2008, Dubrovnik, Croatia, The Design Society.

Conway, A. P. and W. J. Ion (2013). "Enhancing the design dialogue: An architecture to document engineering design activities." Journal of Engineering Design 24(2): 140-164. 